\title{
Aneurisma de la arteria pulmonar en Enfermedad de Behçet
}

\author{
DANIEL G. FERNÁNDEZ A. ${ }^{1}$, CRISTIAN FABIÁN FLÓREZ S. ${ }^{2}$, \\ ALIRIO BASTIDAS ${ }^{2}$, JUAN MANUEL BELLO ${ }^{1}$, RAFAEL VALLE O. ${ }^{1}$, \\ JOHN LONDOÑO P. ${ }^{1}$, ELSA REYES S. ${ }^{1}$, PEDRO SANTOS M. ${ }^{1}$
}

'Servicio de Reumatología e Inmunología. ${ }^{2}$ Servicio de Medicina Interna. Hospital Militar CentralUniversidad Militar Nueva Granada. Bogotá, Colombia.

Recibido el 20 de abril de 2009, aceptado el 9 de noviembre de 2009

El presente trabajo fue financiado en su totalidad por los autores. No requirió financiación externa. Correspondencia a: Daniel G. Fernández Ávila. Teléfono celular: 3204945517 Dirección: Calle 64 No. 52-53 Torre 1 Apartamento 304 Bogotá, Colombia

E-mail: danielfernandezmd@ gmail.com

\section{Pulmonary artery aneurism in a patient with Behçet disease. Report of one case}

Behçet disease is a systemic disease with diverse clinical symptoms which vary according to the organs and systems involved. Typically, patients have oral and genital ulcers and episodic ocular involvement with periods of clinical improvement. We report a 18 year-old male presenting with chest pain and hemoptysis and a history of ulcers in the oral cavity and scrotum. A chest CAT scan showed a mass located adjacent to the heart that a pulmonary arteriography defined as a left pulmonary artery aneurism. The patient was operated, excising left inferior pulmonary lobe. Due to skin and ocular involvement, the patient complied with criteria for Behçet disease. Immunosuppressive treatment with prednisone and azathioprine was started and the patient was discharged from the hospital.

(Rev Med Chile 2010; 138: 82-87).

Key words: Aneurysm; Behçet's Syndrome; Pulmonary artery.
L a enfermedad de Behçet (EB), descrita por primera vez en 1937 por el dermatólogo turco Hulusi Behçet, es una enfermedad crónica sistémica compleja de causa desconocida, caracterizada por la aparición recurrente de úlceras orales y genitales, uveítis a repetición y compromiso variado de distintos órganos o sistemas (piel, mucosas, articular, neurológico, renal, pulmonar, vascular e intestinal $)^{1}$. Esta patología es usualmente vista en los países que se extienden del Mediterráneo hasta el lejano oriente, en lo que corresponde a la ancestralmente conocida ruta de la seda ${ }^{2}$. Su etiopatogenia es desconocida, aunque el concepto más aceptado actualmente como desencadenante de la enfermedad propone que ciertos antígenos de agentes infecciosos (posiblemente por mimetismo molecular), pueden desencadenar una respuesta autoinmune en pacientes genéticamente susceptibles ${ }^{3}$. El presente caso es de particular relevancia, no sólo por reunir las principales características clínicas de esta entidad, sino también por la presencia de una complicación vascular a nivel pulmonar, que puso en riesgo la vida del paciente al momento de la presentación inicial del cuadro clínico.

\section{Caso Clínico}

Paciente de 18 años, sexo masculino, quien presentaba cuadro clínico de 3 meses de evolución consistente en dolor torácico en el hemitórax izquierdo, de características pleuríticas, asociado a episodios de hemoptisis. Los antecedentes personales y familiares eran negativos. A la revisión por sistemas refería que desde hace 2 años presentaba la aparición intermitente de lesiones ulceradas en mucosa oral y escroto, dolorosas, que duraban aproximadamente 2 semanas y resolvían en forma espontánea, para las que no había buscado asistencia médica previamente. Consultó inicialmente 
Aneurisma de la arteria pulmonar en Enfermedad de Behçet - D.G. Fernández A. et al

a hospital local de su región, en el que realizaron estudios iniciales con radiografía de tórax y baciloscopias, los que fueron informados como normales. Por persistencia de la sintomatología, 3 semanas después consultó nuevamente con el cuadro descrito de tos y hemoptisis. Se realizó en su hospital local nuevo estudio imagenológico, con radiografía de tórax que evidenció una imagen radio opaca paracardíaca izquierda (Figura 1), por lo que fue enviado al Hospital Militar Central para continuar estudios complementarios.

En el examen físico de ingreso, se encontraba con signos vitales: FC 98 FR 18 PA 115/70 T $37,1^{\circ} \mathrm{C}$, con palidez mucocutánea significativa, mucosa oral húmeda. A nivel cardiopulmonar: taquicardia, ruidos cardíacos normales sin soplos, ruidos respiratorios disminuidos en base de hemitórax izquierdo. Llamaba la atención la presencia de lesiones descritas en la revisión por sistemas, con 2 úlceras de $0,5 \mathrm{~cm}$ en mucosa oral de labio inferior y una úlcera de $1 \mathrm{~cm}$ de diámetro a nivel del escroto del testículo derecho (Figuras 2 y 3 ). El resto del examen físico se encontraba dentro de límites normales.

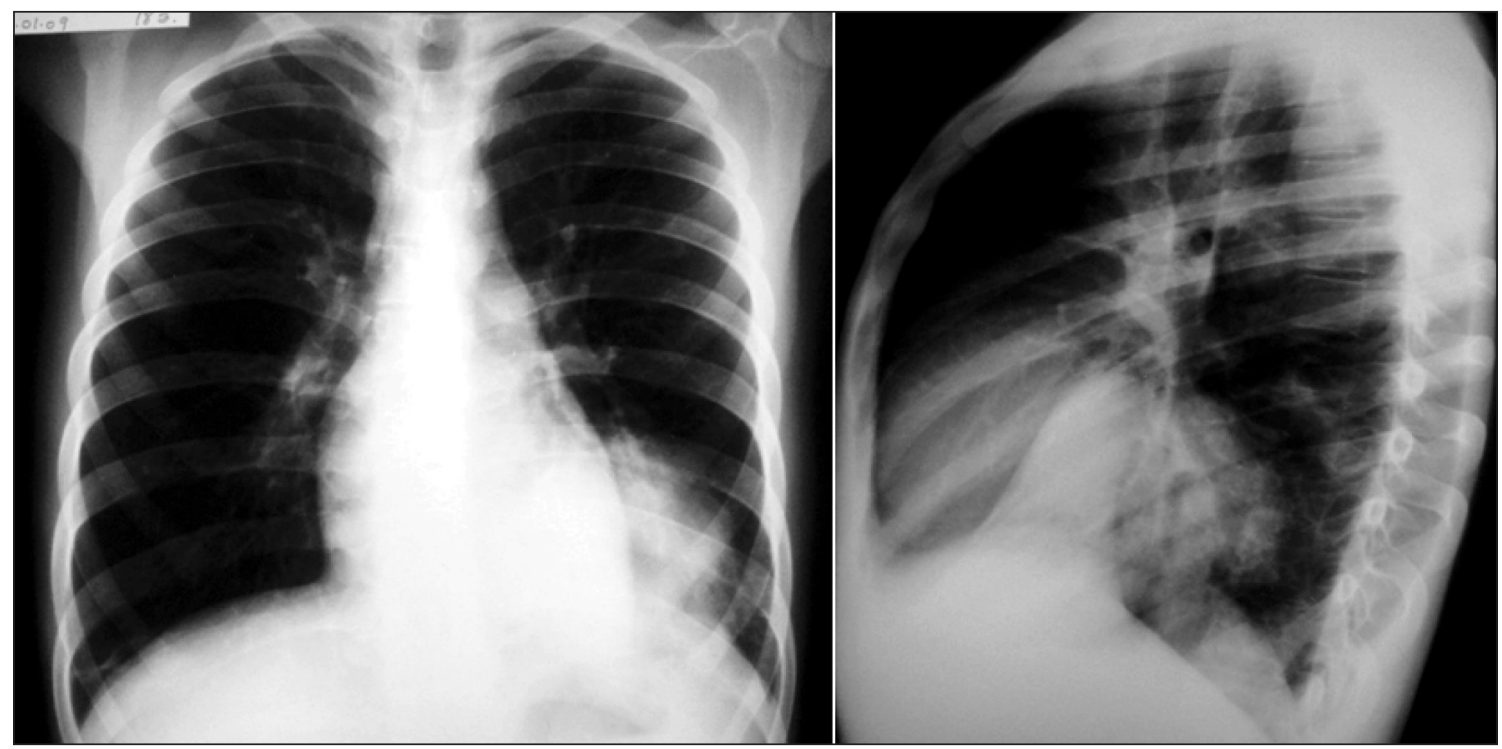

Figura 1. Radiografía de tórax que evidencia una imagen radio opaca paracardíaca izquierda.

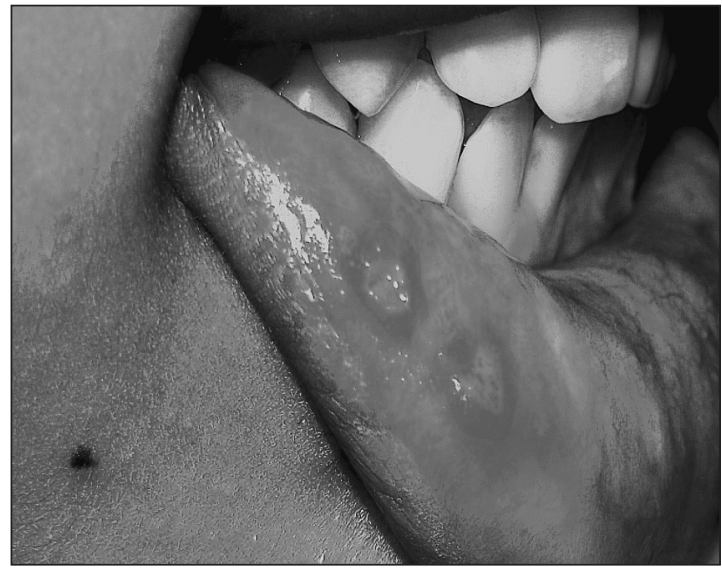

Figura 2. Úlceras orales.

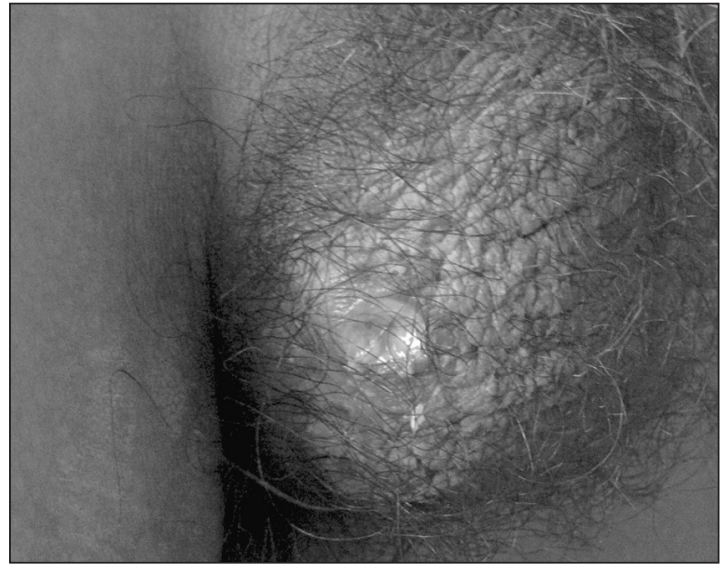

Figura 3. Úlcera genital en escroto. 
Dentro de los estudios iniciales se encontró un hemograma con anemia microcítica heterogénea (Hb 6,3 VCM 76 RDW 18), sin alteración en otras líneas celulares. Adicionalmente, gases arteriales y electrolitos del plasma dentro de límites normales. Se decidió estudio de la imagen encontrada en la radiografía de tórax con una tomografía axial computarizada, la cual evidenció una masa paracardíaca izquierda, de bordes bien definidos con densidad de tejidos blandos que medía aproximadamente $8 \times 10 \times 6$ centímetros (Figura 4).

Con la sospecha de lesión vascular se decidió llevar a arteriografía pulmonar la cual mostró un aneurisma de la arteria pulmonar izquierda que se extendía a la arteria para el lóbulo inferior (Figura 5).

Se realizó en ese instante embolización de la lesión y posteriormente fue llevado a cirugía, encontrándose un aneurisma de la arteria pulmonar izquierda rama para el lóbulo inferior, con trombo en su interior (Figura 6) realizándose lobectomía del lóbulo pulmonar inferior izquierdo.

Por el compromiso de mucosas (úlceras orales y en escroto), con la presencia de la lesión aneurismática descrita, se consideró como posibilidad diagnóstica enfermedad de Behçet, por lo que se realizó prueba de patergia, la cual fue positiva (Figura 7).

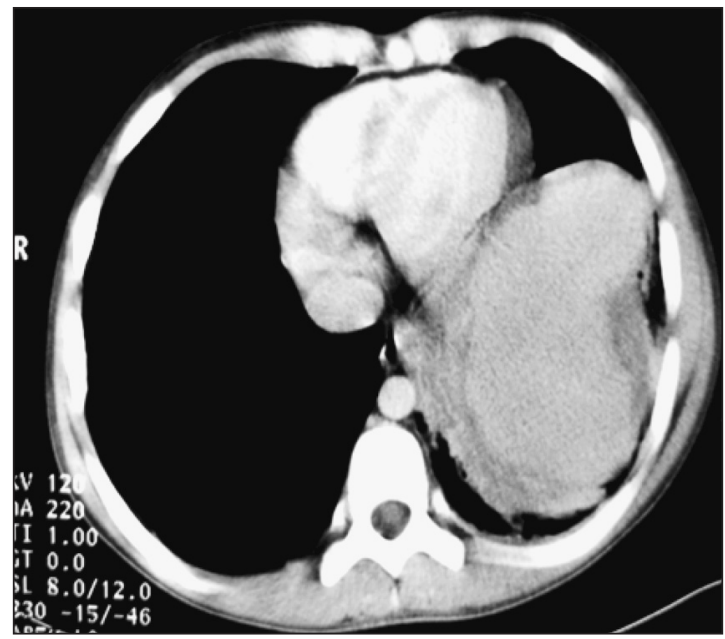

Figura 4. TAC de tórax con masa paracardíaca izquierda.
Se realizó evaluación oftalmológica, con valoración con lámpara de hendidura (Figura 8) la cual evidenció la presencia de sinequias pupilares posteriores (flechas verdes) y pigmento en la cápsula anterior del cristalino (flechas azules), hallazgos que apoyaron el diagnóstico de enfermedad de Behçet.

El perfil inmunológico del paciente muestra ANAS y ANCAS negativos con tipificación de HLA positivo para A24, A26, B27 y B35. Se decidió manejo inmunosupresor con esteroides (prednisona $1 \mathrm{mg} / \mathrm{kg}$ día) y azatioprina. La evolución clínica fue favorable, por lo que se decidió continuar manejo ambulatorio por los servicios de reumatología, cirugía de tórax y oftalmología.

\section{Discusión}

La edad promedio de aparición de la EB es entre los 20 y los 40 años de edad, con igual proporción en el compromiso de ambos géneros, a diferencia de la mayoría de enfermedades autoinmunes en las que existe un mayor número de casos en el sexo femenino ${ }^{4-6}$. La distribución geográfica de la EB es peculiar. El país con mayor prevalencia es Turquía (80-421 por 100.000 habitantes $)^{7-9}$. Además se encuentra una alta prevalencia en otros países del mediano y lejano

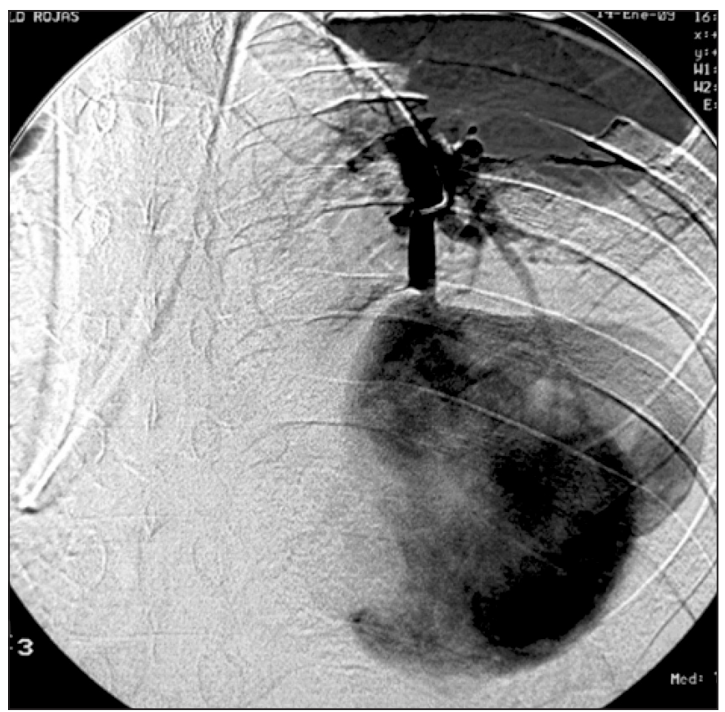

Figura 5. Arteriografia que evidencia aneurisma de la arteria pulmonar izquierda. 


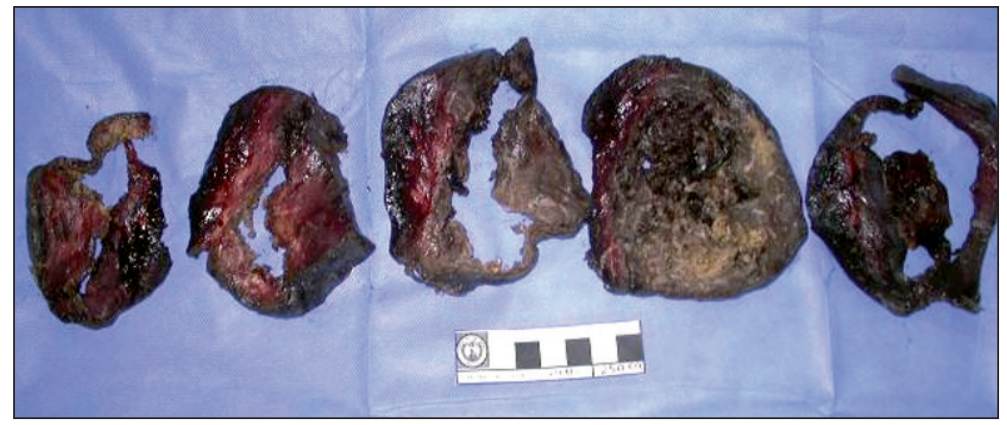

Figura 6. Aneurisma de la arteria pulmonar izquierda con trombo en su interior.

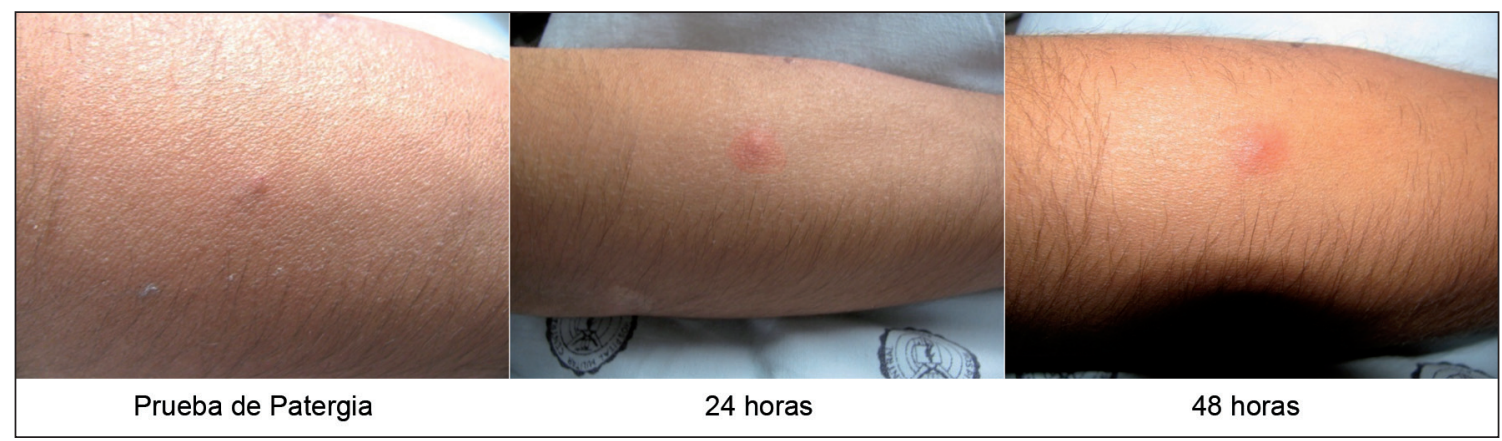

Figura 7. Prueba de patergia.

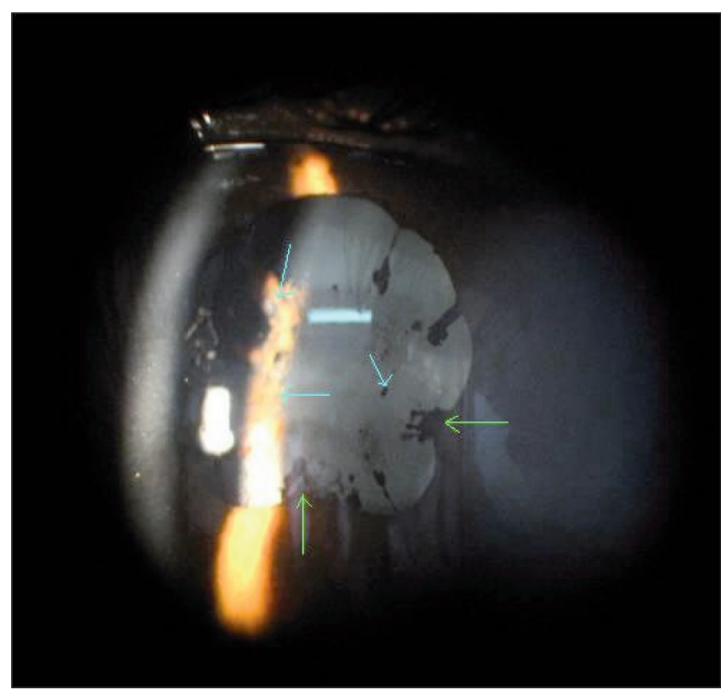

Figura 8. Sinequias pupilares posteriores (flechas verdes). y pigmento en la cápsula anterior del cristalino (flechas azules). oriente como Iraq (17 por 100.000 habitantes $)^{10}$. Su presencia en el hemisferio occidental es baja, con datos del Reino Unido que informan una prevalencia de 0,64 por 100.000 habitantes, España con 6,4 casos por 100.000 y Estados Unidos con 8,6 por $100.000^{4}$.

En cuanto al cuadro clínico de la EB, cabe anotar que es muy variado pues se trata de una enfermedad sistémica que puede llegar a comprometer una gran variedad de órganos y sistemas. Entre los principales hallazgos cabe citar:

Úlceras orales: Son el principal hallazgo clínico (97-99\%) y usualmente el primer síntoma de la enfermedad, precediendo a las otras manifestaciones incluso por varios años ${ }^{4}$. Generalmente, se trata de úlceras menores $(<10 \mathrm{~mm}$ de diámetro) y con menos frecuencia se observan $>10 \mathrm{~mm}$ o de características herpetiformes. 
Úlceras genitales: Son otro hallazgo común (85\%) en la EB. En los hombres usualmente aparecen a nivel del escroto y con mucha menor frecuencia comprometen el pene. Son morfológicamente similares a las úlceras orales y al igual que estas son dolorosas. En las mujeres comprometen los labios mayores y menores.

Compromiso de piel: Se observa en $85 \%$ de los pacientes, usualmente en forma de lesiones pápulo pustulosas, de características morfológicas muy similares al acné, pero a diferencia de este con una distribución preferente hacia las extremidades ${ }^{11}$. Se puede encontrar otro tipo de compromiso en piel como lesiones nodulares, zonas de tromboflebitis superficial, ulceración extragenital y lesiones vasculíticas $^{12}$. La prueba de patergia se incluye dentro del compromiso en piel. Esta prueba, que hace parte de los criterios diagnósticos de EB, es un test de hiperreactividad no específico de la piel. Se induce al pinchar la piel con una aguja estéril, penetrando hasta el tejido celular subcutáneo, usualmente en la cara anterior del antebrazo. Si hay aparición de una pápula 48 horas después de la prueba, se considera positiva.

Compromiso ocular: Presente en 50\% de los pacientes. Es más común y severo en hombres. Usualmente es bilateral y ocurre en los primeros 2 a 3 años de la enfermedad ${ }^{13}$. Generalmente se presenta como una panuveítis crónica bilateral recidivante. Los ataques recurrentes pueden resultar en cambios estructurales como sinequias y cicatrices retinianas.

Compromiso articular: Observado en 30 a 50\% de los casos. Puede presentarse como artritis o artralgias, con compromiso mono u oligoarticular. Usualmente con ubicación periférica sin llegar a producir erosiones ni deformidad.

Compromiso vascular: Pueden comprometerse venas y arterias. Se puede observar como tromboflebitis superficial, trombosis venosa profunda (TVP) o arteritis con formación de aneurismas. A pesar de la presencia de TVP los episodios de tromboembolismo pulmonar son escasos, dado que la presencia de trombos es secundaria al enorme proceso inflamatorio en la pared del vaso ${ }^{14}$. Incluso los expertos recomiendan el manejo de estos episodios de TVP con inmunosupresores y no con anticoagulantes ${ }^{15}$. Los aneurismas pulmonares son el segundo sitio de compromiso arterial en orden de frecuencia, solamente superados por los aneurismas de la aorta ${ }^{16}$. Usualmente afectan a pacientes jóvenes quienes presentan como síntoma más común la hemoptisis. La localización más frecuente es en las arterias del lóbulo inferior derecho, seguido por las arterias pulmonares derecha e izquierda ${ }^{17}$. La más alta mortalidad en enfermedad de Behçet es vista en pacientes con aneurismas de arteria pulmonar ${ }^{18-20}$.

Compromiso de sistema nervioso central (CNS): Ocurre en $5 \%$ de los pacientes y usualmente se presenta en pacientes de género masculino. Se puede observar compromiso parenquimatoso (usualmente en tallo cerebral) o no parenquimatoso (trombosis de senos durales, meningitis ascéptica y vasculitis arterial $)^{21}$.

Los criterios de diagnóstico de la EB se anotan en la Tabla 1.

\section{Tabla 1. Criterios de clasificación de enfermedad} de Behçet

\section{Úlceras orales recidivantes}

Aftas menores, aftas mayores o úlceras herpetiformes observadas por el médico o el paciente, con un mínimo de tres episodios durante un período de 12 meses

\section{Úlceras genitales recidivantes}

Úlceras o cicatrices aftosas observadas por el médico o paciente

\section{Lesiones oculares}

Uveítis anterior y posterior, presencia de células en el vítreo al examen con lámpara de hendidura, o bien, vasculitis retiniana diagnosticada por el oftalmólogo

\section{Lesiones cutáneas}

Eritema nodoso observado por el médico o el paciente, psudofoliculitis o lesiones pápulo-pustulosas; o nódulos acneiformes observados por el médico en pacientes post adolescentes no tratados con glucocorticoides

Positividad de la prueba de hiperreactividad cutánea (Prueba de Patergia)

Evaluado por un médico a las 48 horas

Referencia 22 
En cuanto al pronóstico de la EB, la mayoría de los pacientes entra en remisión completa con el paso de los años. Los desenlaces fatales usualmente están relacionados con compromiso neurológico y formación de aneurismas de arterias pulmonares con sangrado masivo fulminante. Este último pudo ser el desenlace de nuestro paciente si no se interviene en forma oportuna.

Dada la naturaleza sistémica de la EB, el tratamiento debe ir enfocado al órgano o sistema comprometido por la enfermedad en un momento dado, asimismo, el manejo varía de acuerdo a la severidad de los síntomas ${ }^{23}$. En general, el manejo médico incluye medicamentos como colchicina, azatioprina, prednisolona, ciclosporina A y ciclofosfamida. Adicionalmente se han empleado medicamentos biológicos como infliximab y etanercept. El tratamiento debe ser multidisciplinario y su complejidad varía de acuerdo a la misma heterogeneidad de las manifestaciones clínicas de la enfermedad, en un espectro que va desde tratamientos tópicos para las manifestaciones mucocutáneas leves hasta pulsos de esteroides o ciclofosfamida para compromiso de SNC.

\section{Referencias}

1. Krause I, Weinberger A. Behçet's disease. Curr Opin Rheumatol 2008; 20: 82-7.

2. Verity DH, Marr JE, Ohno S, Wallace GR, Stanford MR. Behçet's disease, the Silk Road and HLA-B51: historical and geographical perspectives. Tissue Antigens 1999; 54: 213-20.

3. Vidaller A, Formiga F, Sais G, Pujol R. Tratado Hispanoamericano de Reumatología. $1^{\text {a }}$ Edición. Editorial Nomos, Colombia 2006; 1133-9.

4. Yurdakul S. Behçet's Syndrome. Best Pract Res Clin Rheumatol 2008 22; 5: 793-809.

5. Houman MH, Neffati H, Braham A, Harzallah O, Khanfir M, Miled M, et al. Behçet's disease in Tunisia Demographic, clinical and genetic aspects in 260 patients. Clin Exp Rheumatol 2007; 25 (Supl 45): S58-S64.

6. Bang D, Yoon KH, Chung HG, Choi EH, Lee ES, Lee S. Epidemiological and clinical features of Behçet's disease in Korea. Yonsei Med J 1997; 38: 428-36.

7. Idil A, Gurler A, Boyvat A, Caliskan D, Ozdemir O, Isik $\mathrm{A}$, et al. The prevalence of Behçet's disease above the age of 10 years. The results of a pilot study conducted at the
Park Primary Health Care Center in Ankara, Turkey. Ophthalmic Epidemiol 2002; 9: 325-31.

8. Azizlerli G, Kose AA, Sarica R, Gul A, Tugal I, Kulac M, et al. Prevalence of Behçet's disease in Istanbul, Turkey. Int J Dermatol 2003; 42: 803-6.

9. Cakir N, Dervis E, Benian O, Pamuk ON, Soumezates $\mathrm{N}$, Rehimoglu R, et al. Prevalence of Behçet's disease in rural western Turkey: a preliminary report. Clin Exp Rheumatol 2004; 22 (Suppl 34): S53-S5.

10. Al-Rawi ZS, Neda AH. Prevalence of Behçet's disease among Iraqis. Adv Exp Med Biol 2003; 528: 37-41.

11. Alpsoy E, Donmez L, Bacanli A, Apaydin C, Butun C. Review of the cronology of clinical manifestations in 60 patients with Behçet's disease. Dermatology 2003; 207: 354-6.

12. Alpsoy E, Zouboulis CC, Ehrlich GE. Mucocutaneous lesions of Behçet's disease. Yonsei Med J 2007; 48: 57385.

13. Marshall SE. Behçet's disease. Best Pract Res Clin Rheumatol 2004 18; 3: 291-311.

14. Stone J. Vasculitis: A collection of pearls and myths. Rheum Dis Clin North Am 2007; 33: 691-739.

15. Kahraman O. Long-term course of deep venous thrombosis in patients with Behçet's disease. Arthritis Rheum 2003; 48 (Suppl): S385-S90.

16. Erkan F, Gul A, Tasali E. Pulmonary manifestations of Behçet's disease. Thorax 2001; 56: 572-8.

17. Tunaci M, Ozkorkmaz B, Tunaci A, Gui A, Engin G, Acunas B. CT findings of Pulmonary arterial aneurisms during treatment for Behçet's disease. Am J Roentgenol 1999; 172: 729-33.

18. Hamuryudan V, Yurdakul S, Moral F, Numan F, Tuzun $\mathrm{H}$, Tuzuner $\mathrm{N}$, et al. Pulmonary arterial aneurisms in Behçet's syndrome: a report of 24 cases. Br J Rheumatol 1994; 33: 48-51.

19. Hamuryudan V, Er T, Seyahi E, Akman C, Tuzun H, Fresko I, et al. Pulmonary arterial aneurisms in Behçet's syndrome. Am J Med 2004; 117: 867-70.

20. Yazici H, Esen F. Mortality in Behçet's syndrome. Clin Exp Rheumatol 2008; 26: s138-40.

21. Ilhan D, Gulcan E, Uzuner N, Celikkas E. Cerebrovascular manifestations of Behçet's disease. J Clin Neurosci 2009; 16 (5): 576-8.

22. International Study Group of Behçet Disease. Criteria for diagnosis of Behçet's disease. Lancet 1990; 335: 1078 80.

23. Seyahi E, Fresko I, Melikoglu M, Yazici H. The management of Behçet disease. Acta Reumatol Port 2006; 31: 125-31. 